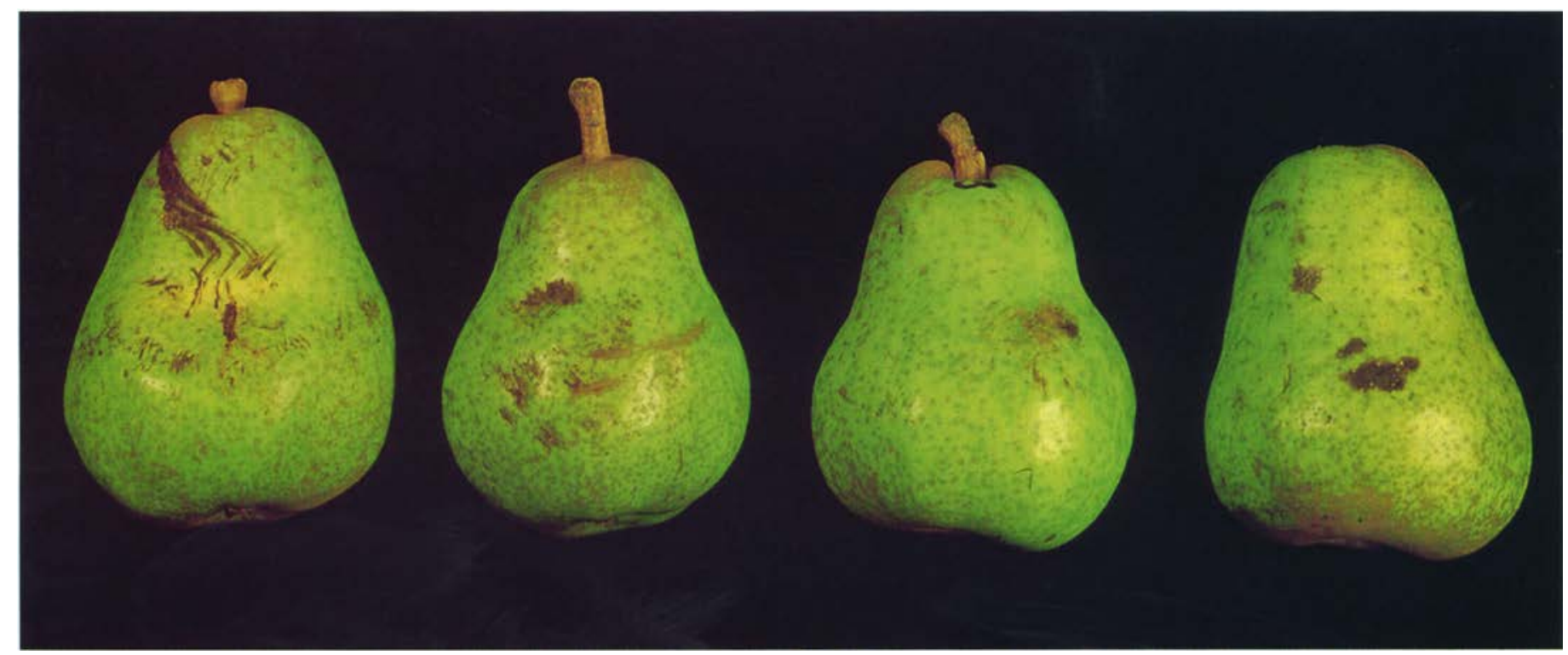

Bartlett pears can be damaged during harvest by pickers or by jostling in the field bin.

\title{
Commercial handling influences quality and ripening of Bartlett pears
}

\author{
I. Tayfun Agar a Elizabeth J. Mitcham
}

\begin{abstract}
Pear fruit injury occurs during harvest and additional injuries occur during postharvest handling in packinghouses. With increased commercial handling, Bartlett pears exhibited higher rates of ethylene production and respiration, which are associated with ripening. Fruit softening and change in skin color from green to yellow occurred faster in pears that were harvested by a commercial crew and then dumped onto the packing line, as compared to pears that were harvested by a commercial crew, without packing-line handling. Immersion dumps used with flotation salts may be less damaging to pears as compared to non-immersion dumps.
\end{abstract}

Bartlett pears are the leading pear variety in the United States, and approximately 294,000 tons, or $60 \%$ of the total U.S. crop, are produced in California.
Large shipments of Bartlett pears from California are distributed within the United States, as well as to international markets such as Canada, Mexico and Brazil. Fresh Bartlett pear fruit in retail markets often show scuffing, bruising, stem punctures and shriveling. Surface marking of pears can be a result of preharvest factors as well as of improper handling during postharvest operations, and is a major reason for rejection and reduced value of pears in markets. During harvest, physical injuries to the fruit often result from striking other fruit or the surfaces of field bins. Even short drops of 3 inches (about $8 \mathrm{~mm}$ ) may cause substantial impact bruising, which makes the fruit less attractive (Mitcham et al. 1994). Cumulative mishandling of the fruit during and after harvest can result in high percentages of fruit damage (table 1; Mitchell 1992a).

Postharvest handling of pears is mechanized to some extent. Fruit are usually hand picked into bags, which are then emptied into field bins for transport to the packing facility. In the packing facility, fruit are dumped, washed, presized to eliminate undersized fruit, sorted to remove blemished and misshapen fruit, and segregated into fresh market and processing products. Fresh market fruit are sized and packed into cartons (Mitchell 1992b). These operations can be a source of significant damage to the fruit. Therefore fruit delivery systems, conveyers, sorting belts and sizers must be designed to minimize fruit injuries. In addition, the height and number of drops on the packing line must be minimized to avoid injuries (Mitchell 1992a).

\begin{tabular}{lc}
$\begin{array}{l}\text { TABLE 1. Cumulative levels of impact bruising } \\
\text { on Bartlett pears during postharvest handling }\end{array}$ \\
\hline \hline Sampling location & Bruised fruit \\
\hline & $\%$ \\
Tree & 0 \\
Picker bag & 14 \\
Field bin & 26 \\
After dump & 38 \\
After size & 82 \\
\hline Source: Mitchell 1992a
\end{tabular}




\section{Where bruising occurs}

Bruising in pears occurs in two forms: roller bruising and impact bruising. Roller bruising can occur when fruit move, rub or vibrate against hard surfaces such as bins, transfer points on the packing line or other fruit. This results in brown areas or bands on the surface of the fruit with little damage to the flesh. Impact bruising typically results from fruit impacts onto hard surfaces (Mitchell 1992b). The incidence and severity of impact bruises and stem punctures increase with increasing height of the drop.

One of the critical areas where impact bruising damage can occur is during rapid fruit unloading from the bins onto either dry dumps or water dumps. With the dry dump, the bin is usually covered with a padded top and rotated. The fruit are delivered onto a conveyor belt from an opening at the bottom of the padded cover. One version of the wet dump also covers the bin with a padded top, inverts the bin and delivers fruit out of the opening in the padded top into a tank of water. The water absorbs much of the energy of impact and rinses the fruit. It is important for the pears to move out of the water tank to a conveyor before the next bin is dumped to reduce fruit-to-fruit impacts.

The other type of water dump is an immersion dump, in which flotation salts are used to allow the pears to float gently out of the immersed bin and onto a conveyor. These dumps submerge the entire bin as it travels along a conveyer, and pumps circulate the dump-tank water to move the freefloating fruit to an elevator, where it is rinsed and transferred to the packing line. For flotation of pears that are heavier than water, salts such as sodium sulfate and sodium lignin sulfonate are added to the dump water to increase its density, so that the fruit can float.

\section{Effects of bruising}

Mechanical injuries, including impact bruising, are of concern because of their detrimental effects on the fruit's appearance and physiology. Postharvest injuries often result in higher fruit respiratory activity and ethylene production, which increases fruit ripening rates and reduces storage life and shelf life. It has been well demonstrated that plants stressed by mechanical damage from abrasion, bruising or punctures produce significant amounts of ethylene within minutes or hours (Yu and Yang 1980). In addition, bruise damage often enhances fruit rot and water loss (Mitcham et al. 1994).

During our studies on Bartlett pear ripening, we have observed that fruit obtained from packinghouses in commercial boxes often ripen without ethylene treatment or cold storage, while fruit carefully harvested and not packed required at least 24 hours of ethylene conditioning or cold storage to ripen. Although pear ripening is often desirable at the retail market, stimulation of ripening of the commercially packed fruit would have a negative impact if fruit is to be cold stored for later sale or processing. The objective of this study was to investigate the effects of postharvest handling of Bartlett pears on fruit respiration, ethylene production and subsequent ripening.

\section{Sampling pears}

We sampled Sacramento-grown Bartlett pears (Pyrus communis L.) at the initiation of commercial harvest, July 24, 1998: (1) directly from the trees (picked by a crew from UC Davis); (2) from bins at the orchard (commercially picked from the same trees as UC-picked); (3) from bins (commercially picked from different trees in the same orchard) after transport 1 mile to the packinghouse (before bin dump); (4) after water dumping of the fruit (after bin dump); and (5) after sorting, sizing (rope sizer) and tight-fill packaging (packed box). All fruit used in this study were in the 175 to $200 \mathrm{~g}$ ( 80 to 90 count) size range and were free of any visible damage. For packing line sampling, marked fruit were positioned at the start of the flow of fruit from a bin through the packing line. We collected fruit from each of the sampling locations in the packing facility after the marker fruit had passed. Fruit were immediately trans-

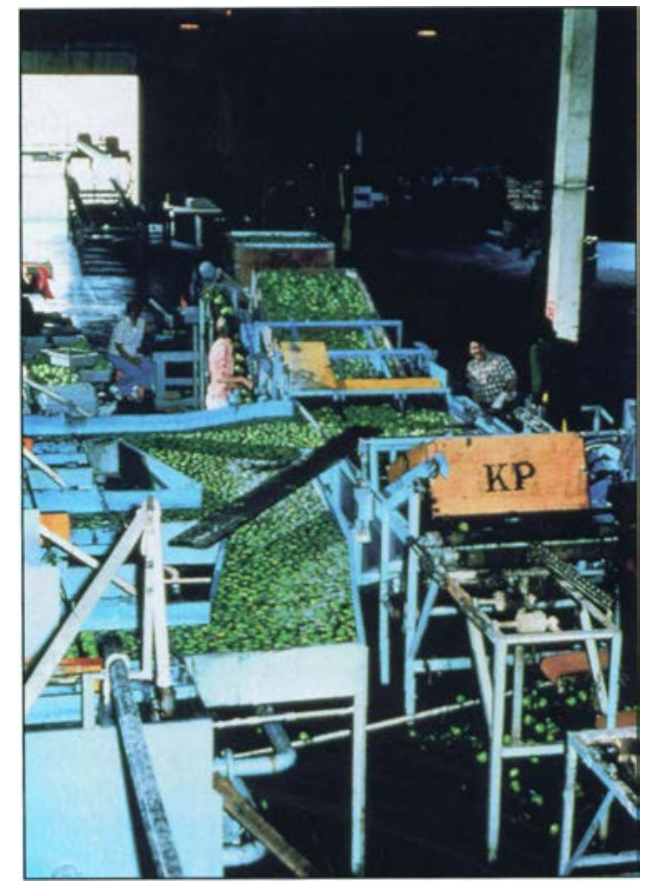

Immersion dumps, used with flotation salts, are least damaging to pears.

ported in an air-conditioned vehicle to the UC Davis Postharvest Pomology Laboratory.

We selected 30 pears (five reps of six fruit) from UC-picked fruit for initial quality evaluation immediately after harvest. In addition, six pears from each location were placed in 1-gallon (3.7 L) glass jars as one replicate. Five replicates were used per sampling location, and all data are reported as the means and SD. Pears were allowed to ripen for 8 days at $68^{\circ} \mathrm{F}\left(20^{\circ} \mathrm{C}\right)$ and then evaluated.

\section{Measuring pear ripening}

We determined flesh firmness at harvest and after 8 days of ripening using an Ametek Penetrometer (Ametek Inc., Matfield, PA) fitted with a $5 / 16$-inch $(8 \mathrm{~mm})$ probe on two sides (with skin removed) of the equatorial region of each pear. External skin color on opposite sides of each fruit was measured with a Minolta Chroma Meter CR-300 in CIE L*a*b* mode under CIE Standard Illuminant $C$. Changes in hue angle $\left(h^{\circ}\right)$, calculated as $h^{\circ}=\arctan$ $b^{*} / a^{*}$ (deg), were used to indicate the color change from green to yellow during ripening. The percentages of decayed pears (with visible mycelial growth) were determined. 


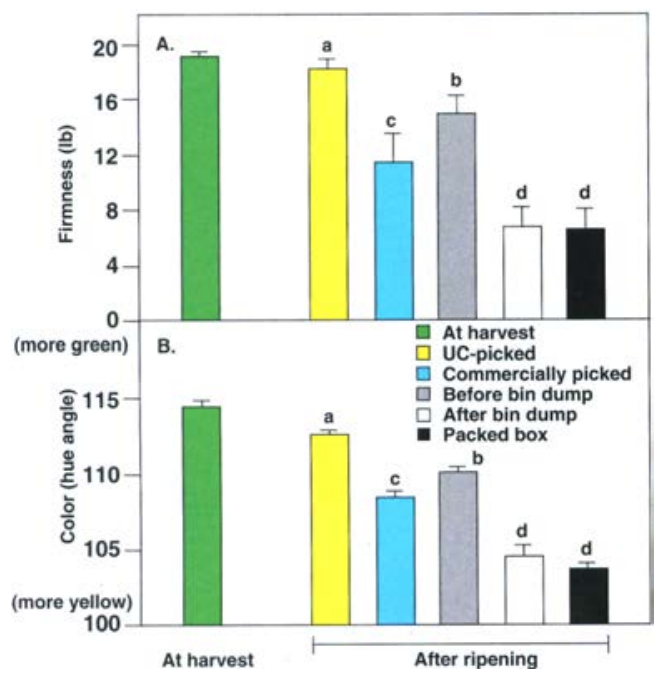

Fig. 1. Firmness (A) and color (B) of Bartlett pears after 8 days of ripening at $68^{\circ} \mathrm{F}\left(20^{\circ} \mathrm{C}\right)$ sampled after harvest or packing. Data points represent the means of five replicates $\pm S D$. The vertical bars represent Duncan's Multiple Range Test for treatment at $P<0.05$. Hue angle attributed to colors as yellow $\left(90^{\circ}\right)$ and green $\left(180^{\circ}\right)$ or an intermediate between any adjacent pair of colors.

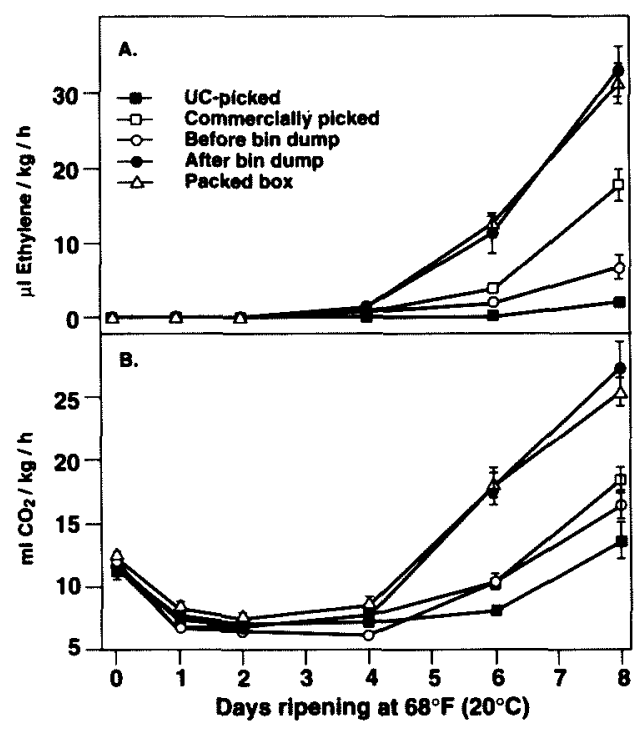

Fig. 2. Changes In ethylene production (A) and respiration rate $(B)$ during ripening of Bartlett pears at $68^{\circ} \mathrm{F}\left(20^{\circ} \mathrm{C}\right)$ sampled after harvest or packing. Data points represent the means of five replicates $\pm S D$.

We measured carbon dioxide and ethylene production rates daily at $68^{\circ} \mathrm{F}$ $\left(20^{\circ} \mathrm{C}\right)$ and standard atmospheric pressure for each replicate and treatment. Six pears (about $1 \mathrm{~kg}$ of fruit) were sealed in a 1-gallon ( $3.7 \mathrm{~L}$ ) jar for 5 to 30 minutes, depending on the ripeness stage, and the headspace was sampled with a $10 \mathrm{~mL}$ syringe. An infrared $\mathrm{CO}_{2}$ analyzer was used to measure $\mathrm{CO}_{2}$. We used a gas chromatograph with FID detector and alumina column to analyze for ethylene.

\section{Statistical analysis}

The experiment consisted of a splitplot design with treatments considered as whole plots and replications randomized within. Data were analyzed by analysis of variance using the general linear model procedure (SAS Institute, Cary, NC), followed by Duncan's Multiple Range Test with a significance level of $P<0.05$.

\section{Pear ripening and decay}

Bartlett pears harvested by university employees exhibited little softening or change in skin color after 8 days at $20^{\circ} \mathrm{C}$ as compared to commercially picked fruit (fig. 1). Commercially picked fruit from the trees were 7 pounds-force softer and less green (higher hue ${ }^{\circ}$ ) after 8 days at $68^{\circ} \mathrm{F}$ $\left(20^{\circ} \mathrm{C}\right)$ than fruit harvested by the UC Davis crew (fig. 1). After 8 days of ripening, fruit sampled after bin dumping or from the tight-fill packed box were 9 pounds-force softer than the same fruit sampled from the harvest bin at the packing facility (fig. 1A). The differences in fruit color followed the same pattern as firmness (fig. 1B). There was no difference in firmness or color between fruit sampled after bin dumping and from the tight-fill box (fig. 1).

There was an inverse relationship between firmness (fig. 1A), and ethylene production and respiration rate (fig. 2). Commercially picked pears exhibited threefold higher ethylene production as compared to UC-picked pears. Pears that were sampled after the bin dump, including the boxed fruit, exhibited the highest ethylene production and respiration rates. The lack of difference in ethylene production and respiration rate between pears sampled immediately after the dump and after box filling may indicate that no additional fruit injury occurred during sorting, sizing and packing. However, because significant damage has been reported from postdump operations (Mitcham et al. 1994; Mitchell 1992a), it seems more likely that wound-induced ethylene and respiration were already maximally induced by the bin dump operation.

All fruit sampled before the bin dump, regardless of harvest operation, exhibited no decay after 8 days at $68^{\circ} \mathrm{F}$ $\left(20^{\circ} \mathrm{C}\right)$ (table 2$)$. In contrast, fruit sampled after the bin dump exhibited $11.5 \%$ decay. Fruit sampled from the packed box exhibited $4.8 \%$ decay, but this was not significantly different from fruit sampled immediately after harvest. The reason that boxed fruit had less decay than fruit sampled immediately after the bin dump is probably because damaged fruit was eliminated during sorting. Most of the decay observed was a result of stem punctures, which probably occurred while fruit were being harvested or dumped. Inoculum in dump tank water may have contaminated fresh wounds and increased the amount of decay.

The delay in ripening of UC-picked fruit was probably a result of very low ethylene production, confirming our previous results (Agar et al. 1999). Mature-green Bartlett pears do not ripen when held at $68^{\circ} \mathrm{F}\left(20^{\circ} \mathrm{C}\right)$ immediately after harvest unless treated with exogenous ethylene. Results of this study clearly demonstrate that rough handling during harvest, and especially during packing, induces wound ethylene production, which triggers ripening of the fruit. Enhanced ethylene production can reduce storage life and shelf life.

In addition, physical damage to the fruit led to a greater percentage of fruit with postharvest decay and reduced the fruit's visual appeal. The results of Mitchell (1992a) clearly dem-

\begin{tabular}{|c|c|}
\hline \multicolumn{2}{|c|}{$\begin{array}{c}\text { TABLE 2. Percent decay of Bartlett pears after } \\
8 \text { days of ripening at } 68^{\circ} \mathrm{F}\left(20^{\circ} \mathrm{C}\right)\end{array}$} \\
\hline Sampling location & Decay \\
\hline $\begin{array}{l}\text { Commercially picked } \\
\text { UC-picked } \\
\text { Before bin dump } \\
\text { After bin dump } \\
\text { Packed box }\end{array}$ & $\begin{array}{c}\% \\
0 \mathrm{~b} \\
0 \mathrm{~b} \\
0 \mathrm{~b} \\
11.5 \mathrm{a} \\
4.8 \mathrm{~b}\end{array}$ \\
\hline \multicolumn{2}{|c|}{$\begin{array}{l}\text { "Data points represent the means of five replicates. } \\
\text { Mean separation was done by Duncan's Multiple } \\
\text { Range Test. Means with the same letters are not } \\
\text { statistically significant at } P<0.05 \text {. }\end{array}$} \\
\hline
\end{tabular}


onstrated that impact bruising of Bartlett pears increases cumulatively from the field bin $(26 \%)$ to after the bin dump (38\%) to after the sizing operation $(82 \%)$ (table 1). Mitchell found that additional damage occurred after the bin dump; namely, after sizing. According to our observations, ethylene production and respiration rates were maximized in pears after the bin dump, and no additional increases occurred between the dump and the packed box in this packinghouse. This was also evidenced by a similar rate of ripening of the fruit collected after the bin dump and from the packed box (fig. 1). Wound ethylene synthesis may have been fully stimulated following the bin dump, and therefore additional handling had no further effect.

These data indicate that significant fruit damage can occur during the dumping operation with a nonimmersion water dump. The immersion water dumps that are commonly used by the apple industry should be less damaging. However, damage from nonimmersion water dumps used in pear packinghouses can be reduced through proper management. The bin should be covered with a wellpadded top during rotation. Fruit should be dumped gradually into the water tank to allow the water to cushion and reduce fruit-to-fruit contact. In addition, chlorine (50 to $100 \mathrm{ppm}$ ) could be added to water tanks to prevent the spread of inoculum and thereby reduce decay, except when sodium lignin sulfonate is used for flotation because it is incompatible with chlorine. When chlorine is used, fruit must be rinsed with fresh water upon removal from the tank to prevent skin damage.

\section{Reducing damage to pears}

Pear fruit injury accumulates during postharvest handling. The ripening rate of pears that were not cold stored or treated with exogenous ethylene was closely associated with wound ethylene production. Bartlett pears exhibited increasing rates of ethylene production and respiration and consequently faster ripening with increased postharvest handling. The harvest operation and nonimmersion wa- ter dump at the packinghouse were significant sources of fruit injury.

Based on previous research with Bartlett pears (Mitchell and Mayer 1985; Mitcham et al. 1994) and our study observations, the following recommendations are made to assist the pear industry to avoid wound ethylene synthesis and to reduce quality problems associated with external damage of pears.

- Use gentler picking containers (padded buckets) to reduce bruising and scuffing.

- Keep plastic bins clean to reduce fruit scuffing.

- Line wooden bins with plastic to significantly reduce fruit scuffing.

- Consider providing incentives to harvest crews to minimize fruit damage.

- Use air-ride suspension when transporting fruit from orchards to packinghouse, especially when travel is over rough roads.

- Use immersion dumps with flotation salts to minimize postharvest damage to pears.

- Reduce damage from nonimmersion water dumps by allowing fruit to move onto conveyors before additional fruit is dumped.

- Avoid dropping pears onto hard surfaces such as hard plastic, wood or supported belts that can be damaging, especially at heights of 6 inches (about $15 \mathrm{~cm}$ ) or greater. Minor modifications, such as adding padding, curtains to slow fruit and suspended belts at fruit transfer points, can minimize damage due to drops and its effects on fruit quality.

I. T. Agar is a visiting scholar from the Department of Horticulture, University of Çukurova, Adana, Turkey, and E.J. Mitcham is a Postharvest Extension Specialist, Pomology Department, UC Davis.

\section{References}

Agar IT, Biasi WV, Mitcham EJ. 1999. Exogenous ethylene accelerates ripening responses in 'Bartlett' pears regardless of maturity or growing region. Postharv Biol and Technol 17:67-78.

Mitcham E, Thompson J, Elkins R, et al. 1994. Development and demonstration of solutions to problem areas identified in postharvest handling of pears. 1994 Reports: Research Projects for California Bartlett pears. California Pear Advisory Board, Sacramento, CA.

Mitchell FG. 1992a. Preparation for fresh market. In: Kader AA (ed.). Postharvest Technology of Horticultural Crops. Oakland, CA: Publication 3311, UC Div Agri and Nat Resources. p 31-8.

Mitchell FG. 1992b. Postharvest handling systems: Temperate zone fruits (pome fruits and stone fruits). In: Kader AA (ed.). Postharvest Technology of Horticultural Crops. Oakland, CA: Publication 3311, UC Div Agri and Nat Resources. p 215-22. Mitchell FG, Mayer G. 1985. Reducing surface injury on pears. California Fruit Grower 62(3):26-8.

Yu Y, Yang SF. 1980. Biosynthesis of wound ethylene. Plant Physiol 66:281-5. 